\title{
Facteurs de risque et caractéristiques cliniques et lésionnelles de la lymphadénite caséeuse ou maladie des abcès chez les ovins au Maroc
}

\author{
Faouzi Kichou ${ }^{1 *}$ Ahmed Mechaal ${ }^{1}$ \\ Mohammed Bouslikhane ${ }^{1}$ Ahlam Kadiri ${ }^{1}$ Khalil Zro ${ }^{2}$ \\ Jaouad Berrada ${ }^{1}$
}

Mots-clés

Ovin, lymphadénite caséeuse, abcès, facteur de risque, histopathologie, épidémiologie, Maroc

Submitted: 27 January 2016 Accepted: 5 September 2016 Published: 26 October 2016

\section{Résumé}

La présente étude a eu pour objectifs d'estimer la prévalence et les facteurs de risques de la lymphadénite caséeuse chez les ovins de la région de l'Oriental au Maroc, et de détérminer ses caractéristiques cliniques et lésionnelles. Des enquêtes ont été réalisées dans différentes provinces de cette région. Elles ont porté sur 6376 animaux répartis dans 107 élevages. De plus, la recherche des lésions, et la collecte des ganglions lymphatiques et des abcès en vue d'un examen histopathologique ont été effectuées dans cinq abattoirs de la région. Les résultats ont montré que pratiquement $100 \%$ des élevages (106 sur 107) étaient affectés par la lymphadénite caséeuse. La prévalence individuelle globale de cette maladie a été de $24 \%$ et les prévalences moyennes au sein des élevages et aux abattoirs ont été respectivement de 28 et $16 \%$. Outre une hygiène défectueuse et une densité animale trop élevée dans les bergeries, I'usage d'équipements traumatisants (mangeoires, abreuvoirs et clôtures métalliques) a semblé constituer le principal facteur favorisant la dissémination des abcès entre animaux d'un même troupeau. Les abcès superficiels, ouverts ou non, avec hypertrophie ganglionnaire satellite, ont été présents chez tous les animaux affectés. Quoique de localisation variable, environ $79 \%$ des lésions se situaient à la tête ou à l'encolure. L'examen histopathologique des ganglions a révélé deux types de lésions : des abcès au stade évolutif ( $87 \%$ ), et des lésions de nécroses et de suppurations diffuses et sévères du tissu lymphoïde (13\%). Dans les poumons la plupart des abcès étaient bien délimités et circonscrits.

- Pour citer cet article : Kichou F., Mechaal A., Bouslikhane M., Kadiri A., Zro K., Berrada J., 2016. Risk factors and clinical and lesion characteristics of caseous lymphadenitis or abscess disease in sheep in Morocco [in French]. Rev. Elev. Med. Vet. Pays Trop., 69 (2): 79-86

\section{INTRODUCTION}

La lymphadénite caséeuse (LC) des ovins, ou maladie des abcès, est due à Corynebacterium pseudotuberculosis, bacille à Gram positif, immobile et aéro-anaérobie. La maladie se caractérise par la formation de pyogranulomes localisés principalement dans les ganglions lymphatiques superficiels, mais aussi dans les ganglions lymphatiques profonds et dans les poumons. Plus rarement, d'autres localisations sont observées comme le foie, les reins, le cœur, le scrotum

1. Département de pathologie et santé publique vétérinaires, Institut agronomique et vétérinaire Hassan II, BP 10101, Rabat, Maroc.

2. Biopharma, Agdal Rabat Maroc.

* Auteur pour la correspondance

Tél. : +212661372357 ; email : f.kichou@iav.ac.ma et les mamelles (Pépin et al., 1988 ; Pépin et al., 1999 ; Pépin, 2003 ; Brugère-Picoux, 2004).

Une contamination précoce des jeunes animaux par leurs mères conduit à des lésions de petite taille pouvant passer inaperçues. Ces lésions évoluent lentement et une expression clinique manifeste n'est observée que chez les adultes. Aussi, le pourcentage d'animaux porteurs d'abcès de grande taille augmente avec l'âge (Moller et al., 2000). La virulence de la maladie est liée à l'expression de la phospholipase D par C. pseudotuberculosis (Pépin, 2003 ; D’afonseca et al., 2008 ; Windsor, 2011).

L'importance de la maladie tient aux pertes économiques qu'elle engendre. En effet, la présence d'abcès superficiels altère la qualité et la valeur commerciale des animaux alors que la présence d'abcès profonds et pulmonaires est souvent associée à un amaigrissement progressif. Les pertes économiques sont dues à la diminution des productions (viande, laine et lait), de l'efficience de la reproduction, 
de la valeur marchande des animaux, à la dévaluation des peaux, et aux saisies partielles ou totales à l'abattoir (Brugère-Picoux, 2004 ; Windsor, 2011).

La $\mathrm{LC}$ a été décrite dans tous les pays où l'élevage ovin est important et le mode d'élevage essentiellement extensif (Pépin et al., 1999 ; Windsor, 2011). Au Maroc, la LC des ovins n'est apparue que vers la fin des années 1980 et, depuis, la maladie a connu une émergence alarmante dans les troupeaux ovins à l'échelle nationale, en particulier dans la région de l'Oriental où les éleveurs se plaignent des pertes qu'elle occasionne. Les conditions environnementales dans ces régions semi-arides à arides seraient propices à l'établissement de l'enzootie chez les petits ruminants. De même, la maladie a été qualifiée de dominante pathologique dans d'autres régions du pays, notamment chez les ovins, les caprins et les camélidés des régions du nord et du sud du Maroc (Ait Balahcen, 2000 ; Kichou et al., 2003 ; Ramiche, 2001). Les pertes liées à cette maladie semblent considérables et peuvent même s'accroître en l'absence d'un plan de lutte. Ainsi, cette étude a eu pour objectif de déterminer la prévalence réelle de LC, ses facteurs de risque et ses caractéristiques cliniques et lésionnelles chez les ovins des provinces de l'Oriental au Maroc.

\section{MATERIEL ET METHODES}

\section{Enquêtes à l'échelle des troupeaux et examens cliniques}

Des enquêtes ont été effectuées dans des élevages ovins de cinq provinces de la région à l'aide d'un questionnaire afin de recueillir les informations relatives aux troupeaux ovins (mode de conduite, antécédents morbides, présence et évolution de la LC). Par la suite, les animaux de chaque troupeau visité ont fait l'objet d'un examen clinique pour détecter la présence d'abcès cutanés et/ou ganglionnaires, et déterminer leurs caractéristiques et leur répartition. Les observations cliniques ont été notées sur une fiche d'examen clinique.

\section{Examen des carcasses et collecte des échantillons à l'abattoir}

Sur la base du volume d'ovins abattus, un abattoir municipal et quatre abattoirs ruraux ont été sélectionnés pour y relever les informations relatives aux effectifs, aux caractéristiques des animaux abattus (espèce, race, sexe et stade physiologique), et à la nature, l'importance et la distribution des abcès identifiés sur les carcasses. Des échantillons représentatifs d'abcès ganglionnaires et tissulaires (42 échantillons) ont été prélevés et placés dans du formol tamponné à $10 \%$ en vue d'un examen histopathologique.

\section{Examens histopathologiques}

Après fixation, les échantillons tissulaires ont été préparés selon les techniques histologiques usuelles dont l'inclusion à la paraffine. Des coupes de $5 \mu \mathrm{m}$ ont été préparées, puis colorées par la technique hémalun-éosine. Les lésions microscopiques observées dans les ganglions et tissus en microscopie optique ont été décrites.

\section{Analyse des données}

Les données recueillies ont été consignées dans un tableur (Excel) et ont fait l'objet d'une analyse descriptive pour le calcul des prévalences et la variance. La comparaison des prévalences moyennes à l'échelle des troupeaux a été conduite à l'aide du test $t$ de Student pour les facteurs suivants : race, type de matériel d'élevage, et densité et hygiène des élevages. La comparaison des proportions (prévalences selon les catégories d'âge) a été effectuée par le test du Chi carré avec le logiciel Epi Info 7.

\section{RESULTATS}

\section{Prévalence de la lymphadénite caséeuse}

Sur la base des examens cliniques, la LC a été diagnostiquée dans la quasi-totalité des troupeaux enquêtés (106 sur 107) avec une prévalence individuelle globale de $24 \%$ (1544/6376 examinés). Au sein des 106 élevages affectés, la prévalence moyenne globale a été de $28 \%$, et a varié de 21,7 à 30,8\% selon les provinces (tableau I) sans qu'il y ait eu une différence significative entre celles-ci (Anova, $\mathrm{p}>0,05$ ).

La prévaence de la LC chez les ovins adultes a été légèrement supérieure à celle estimée chez les jeunes (24,8 vs $23,5 \%)$ mais la différence n'était pas significative (test du Chi carré, p > 0,01) (tableau II).

\section{Localisation et distribution des lésions}

Les lésions observées étaient essentiellement des hypertrophies des ganglions lymphatiques, et des abcès ganglionnaires et sous-cutanés. Les localisations les plus fréquentes étaient la tête et la région préscapulaire (79\% des cas) (figure 1). Les abcès pouvaient être uniques ou multiples chez un même animal. L'incision de ces abcès laissait s'écouler un pus épais de couleur crème à jaunâtre (figure 2).

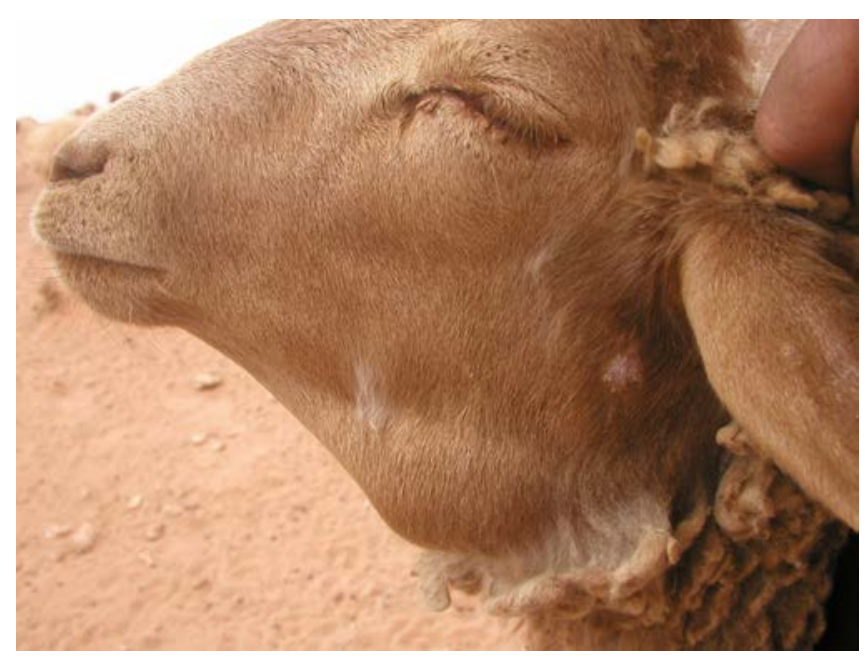

Figure 1 : hypertrophie très marquée des ganglions sous-maxillaire et parotidien chez un bélier de race Beni Guil dans la région de l'Oriental au Maroc (C Kichou, 2013).

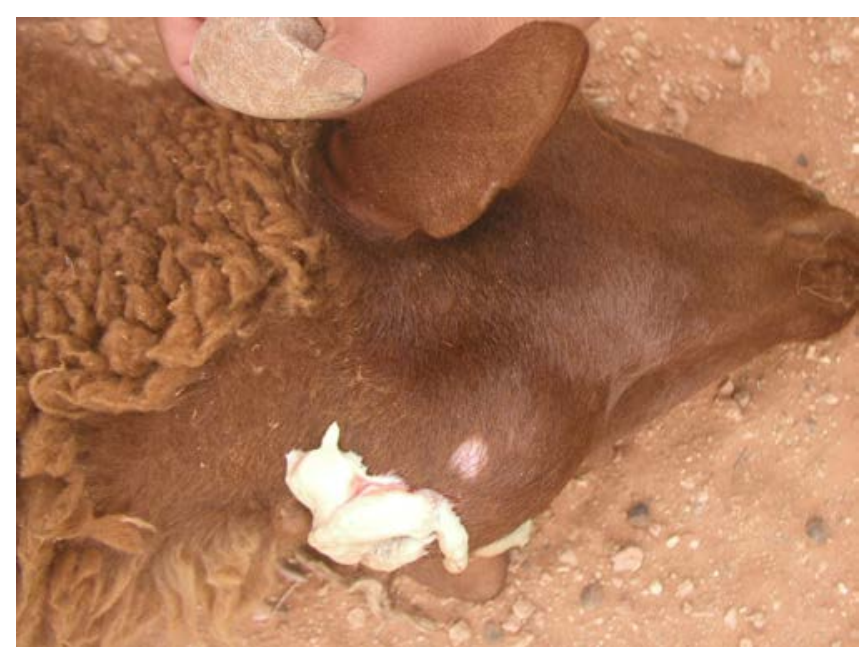

Figure 2 : abcès ouvert du ganglion sous-maxilaire laissant couler un pus épais et abondant chez un bélier de race Beni Guil dans la région de l'Oriental au Maroc (C Kichou, 2013). 


\section{Tableau I}

Prévalences d'ovins porteurs d'abcès de lymphadénite caséeuse dans les élevages selon la province de l'Oriental au Maroc

\begin{tabular}{lcccccrr} 
Province & $\begin{array}{c}\text { Nb. d'élevages } \\
\text { affectés }\end{array}$ & Effectif total & $\begin{array}{c}\text { Nb. d'ovins } \\
\text { affectés }\end{array}$ & $\begin{array}{c}\text { Prévalence } \\
\text { moyenne }\end{array}$ & IC à 95 \% & Min. & Max. \\
\hline Berkane & 7 & 525 & 147 & 29,0 & $23,7-34,3$ & 23,8 & 37,8 \\
Figuig & 17 & 1461 & 321 & 21,7 & $17,3-26,2$ & 7,7 & 35,6 \\
Jerrada & 32 & 1841 & 406 & 27,8 & $23,2-32,3$ & 5,8 & 56,7 \\
Oujda & 45 & 2086 & 566 & 30,8 & $25,8-35,8$ & 9,1 & 96,3 \\
Taourite & 5 & 428 & 104 & 24,2 & $19,0-29,4$ & 17,9 & 28,1 \\
\hline Total & 106 & - & - & 28,0 & $25,4-30,7$ & 5,8 & 96,3
\end{tabular}

IC : intervalle de confiance

\section{Tableau II}

Prévalence de la lymphadénite caséeuse selon la classe d'âge dans les élevages ovins de I'Oriental au Maroc

\begin{tabular}{|c|c|c|c|c|c|c|}
\hline \multirow[t]{2}{*}{$\begin{array}{l}\text { Classe } \\
\text { d'âge }\end{array}$} & \multicolumn{2}{|c|}{$\begin{array}{c}\mathrm{Nb} \text {. } \\
\text { d'ovins }\end{array}$} & \multirow[t]{2}{*}{$\begin{array}{l}\text { Prévalence } \\
\text { estimée }\end{array}$} & \multirow[t]{2}{*}{$\mathrm{Chi}^{2}$} & \multirow[t]{2}{*}{ dl } & \multirow[t]{2}{*}{$\mathbf{p}$} \\
\hline & Examinés & Affectés & & & & \\
\hline Jeune & 2246 & 527 & 23,45 & & & 0,22 \\
\hline Adulte & 4095 & 1017 & 24,84 & 1,48 & 1 & $(>0,05 ; N S)$ \\
\hline
\end{tabular}

dl : degré de liberté ; NS : non significatif

\section{Facteurs de risque liés à la lymphadénite caséeuse}

Les résultats de la comparaison des prévalences moyennes ont montré que les facteurs de risque associés à une forte prévalence de la maladie étaient la densité élevée des animaux dans les bergeries, l'utilisation d'un matériel traumatisant, notamment les abreuvoirs métalliques, et l'absence de mesures d'isolement des animaux malades (tableau III). Les moutons de race pure Beni Guil étaient répartis dans presque la totalité des troupeaux des provinces de Jerrada et de Figuig. Les troupeaux où cette race était présente semblaient avoir une prévalence inférieure $(25,2 \%)$ à celles des troupeaux de races croisées ou mixtes (présence au moins de deux races différentes). Toutefois, la différence des prévalences n'était pas significative.

\section{Tableau III}

Facteurs de risque pour la lymphadénite caséeuse dans les élevages ovins de l'Oriental au Maroc

\begin{tabular}{|c|c|c|c|c|c|c|c|}
\hline Facteur & & Prévalence moyenne & Nb. & $\mathbf{t}$ & dl & $\mathbf{p}$ & \\
\hline \multirow[t]{3}{*}{ Race } & Mixte & 32,20 & 16 & 0,73 & 57 & $>0,1$ & NS \\
\hline & Race croisée & 28,95 & 43 & 1,48 & 89 & $>0,05$ & NS \\
\hline & Race pure & 25,20 & 48 & 1,63 & 62 & $>0,05$ & NS \\
\hline \multirow[t]{2}{*}{ Mangeoire } & Traumatisant & 27,66 & 42 & \multirow{2}{*}{0,06} & \multirow{2}{*}{105} & \multirow{2}{*}{$>0,1$} & \multirow{2}{*}{ NS } \\
\hline & Non traumatisant & 27,82 & 65 & & & & \\
\hline \multirow[t]{2}{*}{ Abreuvoir } & Traumatisant & 29,47 & 77 & \multirow{2}{*}{2,02} & \multirow{2}{*}{105} & \multirow{2}{*}{$<0,05$} & \multirow{2}{*}{+} \\
\hline & Non traumatisant & 23,34 & 30 & & & & \\
\hline \multirow[t]{2}{*}{ Nature de la clôture } & Traumatisant & 29,45 & 16 & \multirow{2}{*}{0,53} & \multirow{2}{*}{105} & \multirow{2}{*}{$>0,05$} & \multirow{2}{*}{ NS } \\
\hline & Non traumatisant & 27,46 & 91 & & & & \\
\hline \multirow[t]{2}{*}{ Densité } & Elevée & 30,36 & 76 & \multirow{2}{*}{3,16} & \multirow{2}{*}{105} & \multirow{2}{*}{$<0,01$} & \multirow{2}{*}{++} \\
\hline & Optimale & 21,36 & 31 & & & & \\
\hline \multirow[t]{2}{*}{ Isolement des ovins affectés } & Absent & 29,80 & 59 & \multirow{2}{*}{1,70} & \multirow{2}{*}{105} & \multirow{2}{*}{$<0,05$} & \multirow{2}{*}{+} \\
\hline & Présent & 25,23 & 48 & & & & \\
\hline \multirow[t]{2}{*}{ Désinfection des locaux } & Absent & 28,46 & 91 & \multirow{2}{*}{1,25} & \multirow{2}{*}{105} & \multirow{2}{*}{$>0,1$} & \multirow{2}{*}{ NS } \\
\hline & Présent & 23,74 & 16 & & & & \\
\hline \multirow[t]{2}{*}{ Vide sanitaire } & Absent & 27,73 & 99 & \multirow{2}{*}{0,05} & \multirow{2}{*}{105} & \multirow{2}{*}{$>0,1$} & \multirow{2}{*}{ NS } \\
\hline & Présent & 28,01 & 8 & & & & \\
\hline
\end{tabular}

$\mathrm{dl}$ : degré de liberté ; NS : non significatif ; + : différence significative ; ++ : différence très significative 


\section{Prévalence globale des abcès à l'abattoir}

Sur 12345 ovins examinés post mortem pendant trois mois, 7,3\% avaient des abcès (902 carcasses). La prévalence moyenne de la maladie détectée par l'observation des carcasses à l'abattoir a été significativement différente selon les provinces (Anova, $\mathrm{p}<001$ ). La prévalence la plus élevée a été observée dans la province de Jerrada $(25,4 \%)$ et la plus faible dans celle d'Oujda (5,9\%) (tableau IV).

\section{Prévalence des abcès en fonction de l'âge et du sexe}

La prévalence de la LC estimée au niveau des abattoirs a été significativement différente $(\mathrm{p}<0,01)$ selon les trois classes d'âge (tableau V) : la plus forte a été de $16,9 \%$ chez les adultes de plus de quatre ans et la plus faible de $6,3 \%$ chez les jeunes de moins d'un an. Les femelles ont été significativement plus affectées par la LC que les mâles avec des prévalences respectives de 9,2 et 5,4\% (tableau VI)

\section{Tableau IV}

Prévalence moyenne des abcès sur les carcasses ovines à I'abattoir dans l'Oriental au Maroc

\begin{tabular}{lrrrrr} 
Province & $\begin{array}{c}\text { Nb. de } \\
\text { visites }\end{array}$ & $\begin{array}{r}\text { Effectif } \\
\text { abattu }\end{array}$ & $\begin{array}{c}\text { Nb. d'ovins } \\
\text { affectés }\end{array}$ & $\begin{array}{c}\text { Prévalence } \\
\text { moyenne }\end{array}$ & IC à 95 \% \\
\hline Jerrada & 16 & 756 & 190 & 25,4 & $17,4-33,4$ \\
$\begin{array}{l}\text { Oujda } \\
\text { Taourirt }\end{array}$ & 15 & 11367 & 654 & 5,9 & $4,3-7,5$ \\
\hline $\begin{array}{l}\text { Prévalence } \\
\text { moyenne } \\
\text { totale }\end{array}$ & 34 & 12345 & $\begin{array}{c}902 \\
(7,3 \%)\end{array}$ & 15,9 & $8,6-23,2$ \\
\hline
\end{tabular}

IC : intervalle de confiance

\section{Tableau V}

Prévalence de la lymphadénite caséeuse par classe d'âge à I'abattoir dans I'Oriental au Maroc

\begin{tabular}{|c|c|c|c|c|c|c|}
\hline \multirow[t]{2}{*}{$\begin{array}{l}\text { Classe } \\
\text { d'âge }\end{array}$} & \multicolumn{2}{|c|}{$\begin{array}{c}\text { Nb. } \\
\text { d'ovins }\end{array}$} & \multirow[t]{2}{*}{$\begin{array}{l}\text { Prévalence } \\
\text { estimée }\end{array}$} & \multirow[t]{2}{*}{$\mathrm{Chi}^{2}$} & \multirow[t]{2}{*}{ dl } & \multirow[t]{2}{*}{ p } \\
\hline & xaminés & Affectés & & & & \\
\hline $0-1$ an & 2672 & 167 & 6,3 & & & \\
\hline $2-3$ ans & 1037 & 88 & 8,5 & 41,9 & 2 & $\begin{array}{c}<0,0001 \\
(++)\end{array}$ \\
\hline$>4$ ans & 272 & 46 & 16,9 & & & \\
\hline
\end{tabular}

$\mathrm{dl}$ : degré de liberté ; ++ : différence très significative

\section{Tableau VI}

Prévalence moyenne de la lymphadénite caséeuse par sexe relevée à l'abattoir dans l'Oriental au Maroc

\begin{tabular}{|c|c|c|c|c|c|c|}
\hline \multirow[t]{2}{*}{ Sexe } & \multicolumn{2}{|c|}{$\begin{array}{c}\text { Nb. } \\
\text { d'ovins }\end{array}$} & \multirow[t]{2}{*}{$\begin{array}{l}\text { Prévalence } \\
\text { estimée }\end{array}$} & \multirow[t]{2}{*}{$\mathrm{Chi}^{2}$} & \multirow[t]{2}{*}{ dl } & \multirow[t]{2}{*}{$p$} \\
\hline & Examinés & Affectés & & & & \\
\hline Femelle & 2302 & 211 & 9,2 & \multirow{2}{*}{19,2} & \multirow{2}{*}{1} & \multirow{2}{*}{$\begin{array}{c}<0,0001 \\
(++)\end{array}$} \\
\hline Mâle & 1674 & 91 & 5,4 & & & \\
\hline
\end{tabular}

$\mathrm{dl}:$ degré de liberté ; ++ : différence très significative

\section{Nature et localisation des lésions observées}

L'examen ante mortem des animaux a montré la présence d'abcès localisés dans le tissu sous-cutané et les ganglions lymphatiques superficiels. L'examen post mortem a révélé de nombreuses lésions superficielles et internes. En superficie, les ganglions lymphatiques étaient volumineux (2-15 cm de diamètre), et l'incision laissait s'écouler un pus épais et dense de couleur jaune verdâtre (figure 3). $\mathrm{Au}$ niveau interne, les poumons et le foie renfermaient des lésions circonscrites (figure 4) contenant un pus dense, caséeux parfois disposé en lamelles circulaires rappelant l'aspect d'un oignon (figure 5). Vingt pour-cent de ces lésions étaient localisées dans les poumons, $7 \%$ dans le foie, et $7 \%$ dans les ganglions mediastinaux et trachéobronchiques (figure 6) dont la majorité a été retrouvée chez des brebis

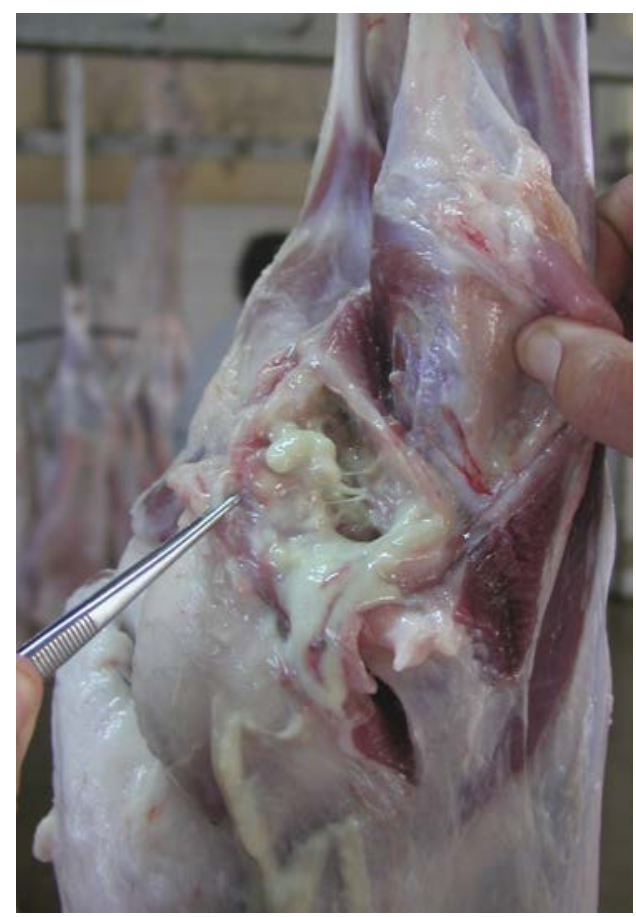

Figure 3 : carcasse de mouton montrant un abcès du ganglion poplité laissant couler un pus crémeux et abondant, inspectée à l'abattoir dans la région de l'Oriental au Maroc (C) Kichou, 2013).

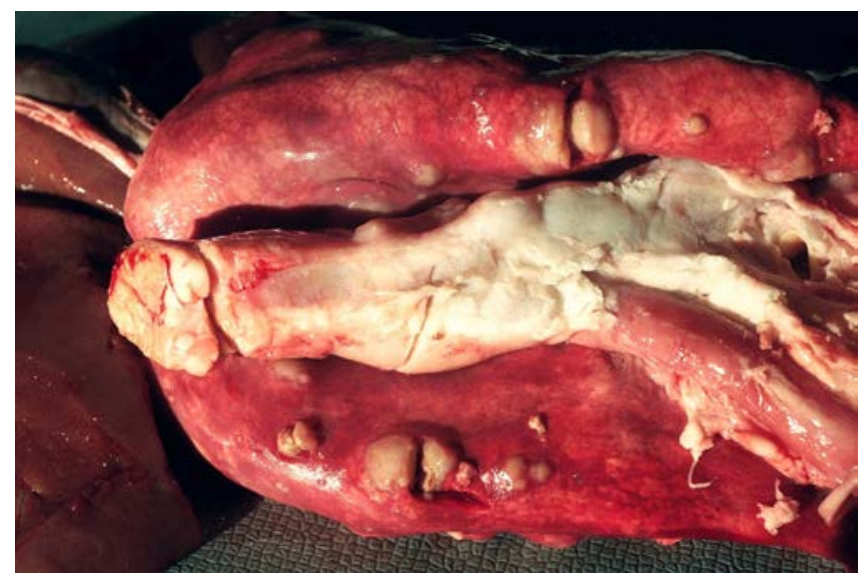

Figure 4 : abcès multiples de taille variable $(0,5-2 \mathrm{~cm}$ de diamètre) sur les poumons d'une brebis inspectée à l'abattoir dans la région de l'Oriental au Maroc (@ Kichou, 2013). 
de plus de deux ans. Parmi celles-ci, environ $80 \%$ avaient des abcès pulmonaires, $13 \%$ avaient des lésions hépatiques et $7 \%$ avaient les deux types de lésions.

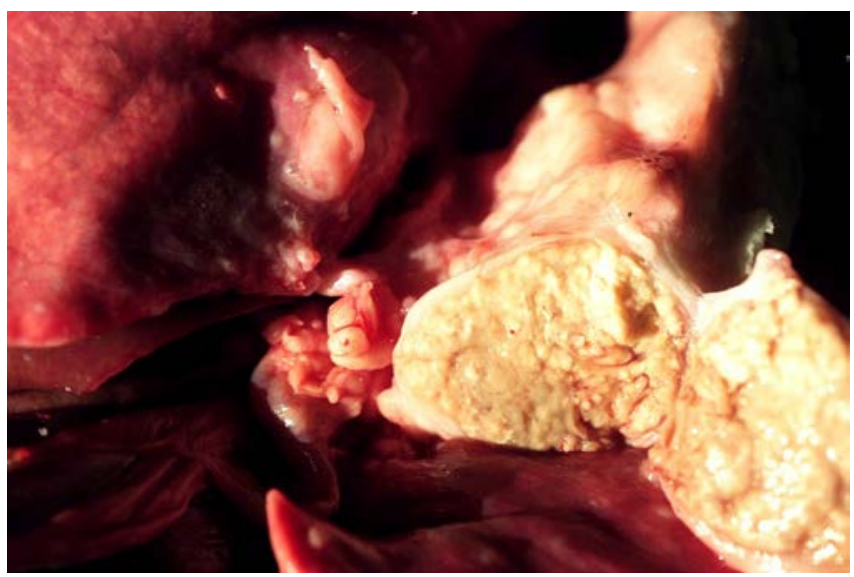

Figure 5 : abcès du ganglion mediastinal chez une brebis (de la figure 4) inspectée à l'abattoir dans la région de l'Oriental au Maroc ; pus dense et sec disposé en grumeaux remplaçant tout le parenchyme lymphoïde (@) Kichou, 2013).

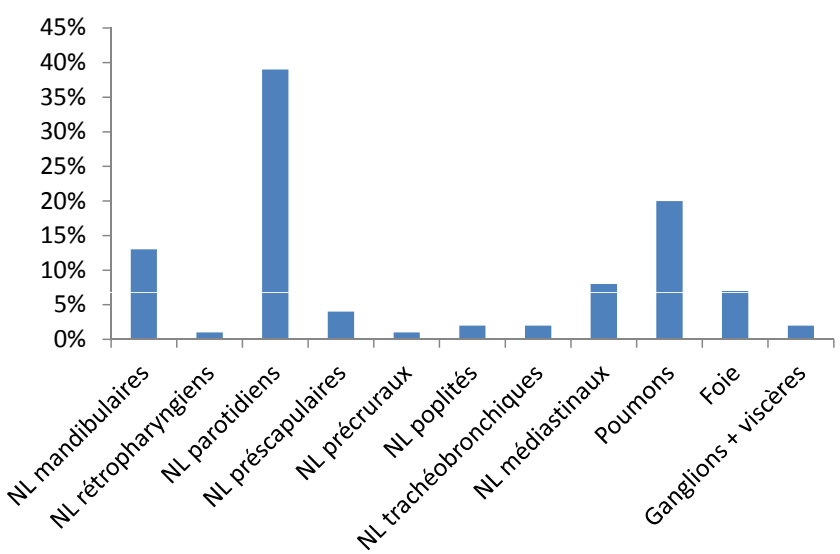

Figure 6 : fréquences de localisation des abcès dans les nœuds lymphatiques (NL) et les viscères de carcasses d'ovins inspectées à l'abattoir dans la région de l'Oriental au Maroc.

\section{Histopathologie}

L'examen histopathologique a concerné les échantillons de ganglions lymphatiques abcédés et/ou d'abcès pulmonaires prélevés sur 15 carcasses ovines.

\section{Lésions ganglionnaires}

Les ganglions lymphatiques ont montré deux types de lésions suppuratives, selon le stade évolutif. Le premier type consistait en des lésions suppurées subaiguës à chroniques, évolutives, sous forme d'abcès unique ou multiples dans $86,7 \%$ des ganglions examinés (figure 7). Ils étaient de taille variable et généralement constitués de trois zones : a) un centre caséo-nécrotique et suppuré (figure 8) contenant des granulocytes neutrophiles, des débris cellulaires nécrotiques et de la fibrine avec parfois un dépôt de calcium ; ce magma occupait la majorité du volume de l'abcès et présentait parfois un arrangement en lamelles plus ou moins distinctes ; b) une couche cellulaire entourant le centre nécrotico-suppuré et constituée de macrophages, de lymphocytes et de plasmocytes ; et c) une couche fibreuse qui tendait à délimiter le processus inflammatoire ; parfois, la fibrose s'étendait de façon diffuse dans le tissu lymphoïde provoquant ainsi un remplacement plus ou moins complet du parenchyme.

Le deuxième type consistait en des lésions aiguës de nécroses et de suppurations diffuses et très sévères du parenchyme lymphoïde folliculaire et parafolliculaire, aboutissant à une destruction massive de ce parenchyme dans $13,4 \%$ des lésions observées.

\section{Lésions pulmonaires}

Les lésions pulmonaires étaient essentiellement des lésions subaiguës à chroniques correspondant à la présence de pyogranulomes multiples évolutifs, similaires à ceux décrits pour les ganglions, mais en général de taille plus petite et beaucoup plus nombreux et plus délimités (figure 9). Ils étaient constitués également de trois zones (figure 10).

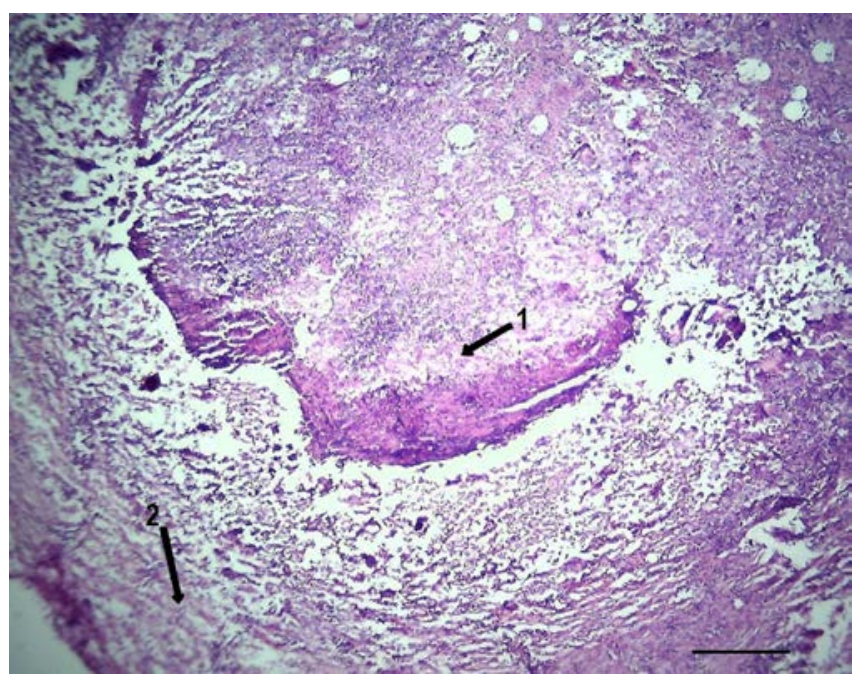

Figure 7 : coupe histologique d'un nœud lymphatique paro tidien dont le tissu lymphoïde folliculaire et parafolliculaire se trouve complètement remplacé par un abcès au centre nécrosuppuré (flèche 1) et entouré d'une coque fibreuse (flèche 2). Hématoxyline et éosine ; bar $330 \mu m$ (@ Kichou, 2015).

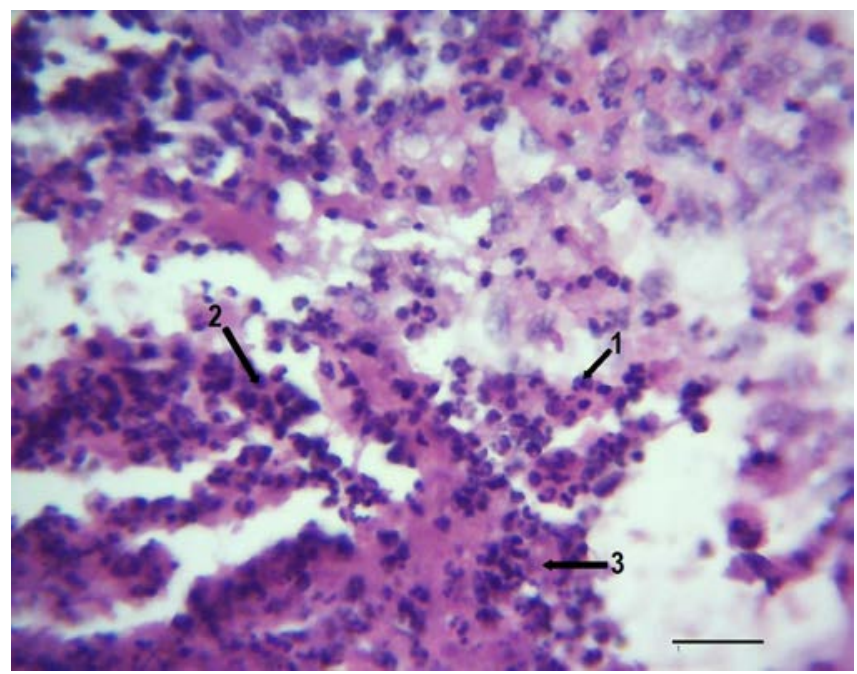

Figure 8 : centre nécro-purulent d'abcès de nœud lymphatique parotidien (de la figure 7) plein de polynucléaires neutrophiles (flèches 1 et 2) et de débris fibrino-nécrotiques (flèche 3). Hématoxyline et éosine; bar 30 m (@ Kichou, 2015). 


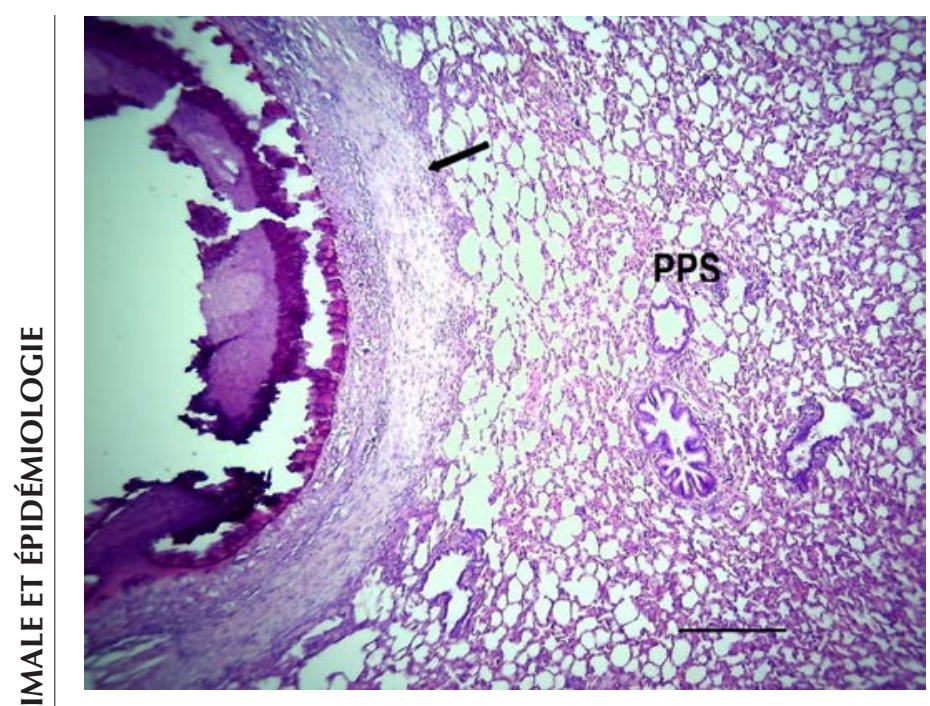

Figure 9 : coupe histologique de poumon de brebis ; pyogranulome (flèche) caractéristique incrusté dans le parenchyme pulmonaire sain (PPS). Hématoxyline et éosine; bar $370 \mu \mathrm{m}$ (C) Kichou, 2015).

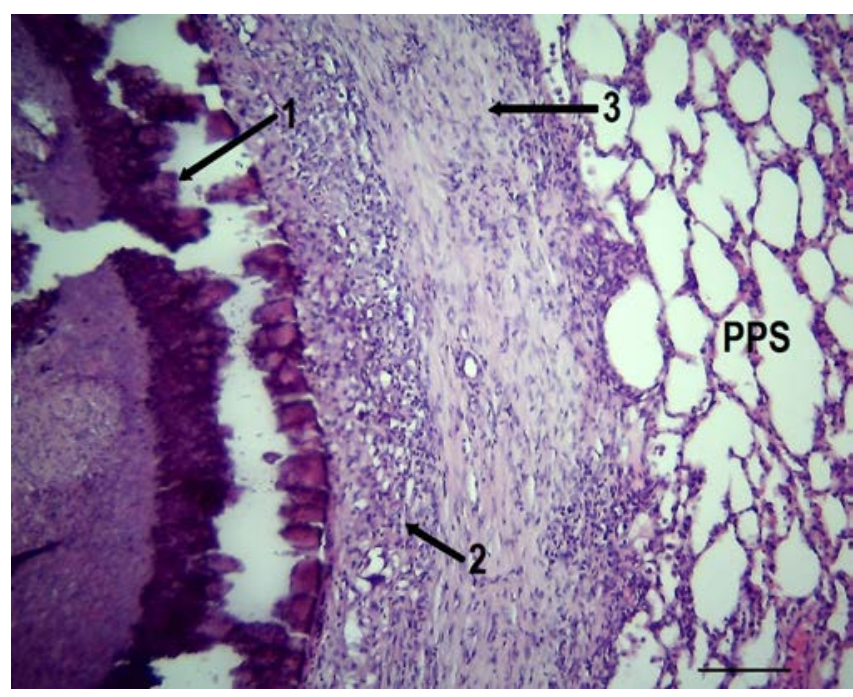

Figure 10 : coupe histologique de poumon de brebis (fort grossissement de la figure 9) ; pyogranulome incrusté dans le parenchyme pulmonaire sain (PPS) et constitué d'un centre nécro-suppuré calcifié (flèche 1), entouré d'une couche lympho-plasmocytaire et macrophagique (flèche 2) et d'une coque fibreuse (flèche 3). Hématoxyline et éosine ; bar $120 \mu \mathrm{m}$ (C) Kichou, 2015).

\section{DISCUSSION}

\section{Anlayse épidémiologique}

Dans la région étudiée, une prévalence individuelle globale de $24 \%$ de la LC a été enregistrée. Aussi, pratiquement $100 \%$ des élevages enquêtés étaient affectés par les abcès cutanés et ganglionnaires. Cette prévalence entre élevages est très élevée, comparée à celles décrites dans d'autres pays, notamment 5-63\% au Royaume-Uni (Baird et Malone, 2005 ; Malone et al., 2005), $80 \%$ en Egypte (Mubarak et al., 1999), et 74-88 \% en Australie occidentale (Pépin et al., 1994 ; Paton et al., 2003).

La prévalence moyenne des abcès estimée au sein des élevages affectés était de $28 \%$ et à l'abattoir de $16 \%$. Ces prévalences sont plus élevées que celles rapportées en Algérie, en Egypte et en Jordanie où elles sont respectivement de 8,9,6,7-19,2 et 15,7\% dans les élevages, et de 0,12,5, et 5,2\% à l'abattoir (Al-Gaabary et al., 2009; Alloui et al., 2009 ; Mubarak et al., 1999 ; Oreiby et al., 2014). La prévalence réelle estimée dans les troupeaux pouvait être plus élevée car, sur les 3976 carcasses inspectées à l'abattoir, 36 \% présentaient uniquement des lésions internes. Cela signifie que la prévalence mesurée dans les élevages était sous-estimée étant donné que seules les lésions externes étaient détectables lors du suivi clinique. Cette situation serait similaire à celle rapportée en Arabie Saoudite (Al-Qarawi, 2005) et en Angleterre (Malone et al., 2005).

La LC provoque des pertes économiques importantes dans l'Oriental. En affectant notamment les moutons engraissés destinés à la fête de l'Aid Al Adha, elle compromet le revenu des éleveurs. En Australie, les pertes se chiffrent en millions de dollars chaque année et résultent d'une diminution de la croissance, de la fertilité, de la production de laine et de lait, ainsi que des pertes dues aux saisies des carcasses et à la dévaluation des peaux (Paton et al., 1994 ; Pépin, 2003 ; Windsor, 2011).

La forte prévalence notée dans la présente étude pourrait s'expliquer par le mode d'élevage essentiellement extensif pratiqué dans la région. Ainsi, les animaux sont exposés à plusieurs facteurs favorisant la transmission et la dissémination de cette infection, comme le partage des parcours par différents troupeaux, et l'existence de plantes et de buissons épineux qui peuvent provoquer des effractions cutanées chez les animaux. D'autres facteurs peuvent également être incriminés, comme l'a montré l'analyse des facteurs de risque, notamment l'utilisation d'abreuvoirs métalliques, la densité élevée d'animaux et le manque d'hygiène. C'étaient, en effet, les facteurs qui prédisposaient le plus à la contamination et à la dissémination des abcès dans les élevages étudiés. Ce même constat a été rapporté par d'autres auteurs (Blood et al., 1994 ; Pépin, 2003 ; Windsor, 2011). Ainsi, toute mesure prophylactique visant à diminuer l'incidence de la maladie devrait $a$ priori passer par la réduction de ces facteurs de risque.

Les enclos ou les bergeries ont des superficies très réduites avec des densités d'animaux très élevées. C'est particulièrement le cas de la province d'Oujda avec une densité de 2,5 têtes $/ \mathrm{m}^{2}$, ce qui augmente le risque de contamination, comme cela a été rapporté par Pépin et al. (1991) et Windsor (2011). Les équipements d'élevage sont souvent traumatisants, ce qui facilite l'inoculation des germes. Aussi, la tonte est un facteur favorisant la contamination à cause des blessures provoquées en l'absence totale de désinfection du matériel et d'antisepsie. Seulement 2 à $6 \%$ des éleveurs isolaient et traitaient les animaux affectés, et $7 \%$ utilisaient le vide sanitaire. Ceci a des conséquences directes sur la persistance et la propagation de la maladie au sein des élevages (Pépin et al., 1991 ; Windsor, 2011). Les abcès ouverts représentent la principale source de matières virulentes, la principale voie de pénétration des bactéries étant tégumentaire (Pépin, 2003).

Une différence significative a été observée entre les prévalences moyennes relevées dans les abattoirs. En revanche la différence n'était pas évidente entre les provinces de l'Oriental en termes de prévalences estimées dans les élevages où le mode de conduite des troupeaux était relativement identique. La différence entre les abattoirs pourrait s'expliquer par le fait que l'abatttoir municipal d'Oujda, grande agglomération urbaine avec la prévalence la plus faible (5,9\%), reçoit des animaux d'autres régions du Maroc moins affectées par la maladie.

Bien que la différence n'ait pas été significative entre les proportions d'animaux jeunes et adultes affectés au niveau des élevages, la prévalence de la maladie a semblé augmenter avec l'âge. En effet, dans les abattoirs, en raison d'une meilleure détection des abcès, les animaux âgés de plus de quatre ans étaient significativement plus affectés $(16,9 \%)$. Cette même tendance a été rapportée par Girones et al. 
(1992) et expliquée par l'exposition répétée à laquelle sont soumis les ovins de cette catégorie, notamment suite à la tonte (Blood et al., 1994 ; Paton et al., 1988).

\section{Aspects cliniques et lésionnels}

Les lésions observées à l'examen clinique étaient essentiellement des hypertrophies ganglionnaires et des abcès sous-cutanés ouverts ou non. Leur incision laissait s'écouler un pus épais jaunâtre à verdâtre, ressemblant à ce qui a été décrit par plusieurs auteurs et, dans certains cas, rappelant l'aspect d'un oignon, caractéristique des abcès de la LC (Batey, 1986 ; Buxton et Fraser, 1984 ; Pépin et al., 1999 ; Sayed et al., 1995).

Ces abcès ont une nette tendance à survenir dans les mêmes régions du corps : tête et région préscapulaire, tête et mamelle, mais aussi tête, flancs et membres. Ceci peut être expliqué par le mode de pénétration des germes à la suite d'effractions cutanées. Les localisations à la tête et dans la région préscapulaire sont les plus fréquemment rencontrées (79\% des élevages), probablement du fait qu'elles sont plus exposées aux traumatismes et par conséquent aux infections, lors de l'abreuvement, de la prise des repas ou des opérations de tonte (mangeoires et abreuvoirs métalliques, et ciseaux de tonte).

La majorité des lésions internes se trouvait dans les poumons (80,7\% des cas), suggérant qu'une trasmission aérienne est possible. Des chiffres comparables ont été rapportés en Arabie Saoudite (AlQarawi, 2005), en Hollande (Shreuder et al., 1986) et en Angleterre (Malone et al., 2005 ; Smith, 1981). En majorité ces abcès étaient d'évolution subaiguë à chronique, et localisés dans les ganglions, les poumons et le foie. Les lésions microscopiques observées étaient caractéristiques des abcès induits par Corynebacterium pseudotuberculosis chez les ovins (Euzeby, 1999 ; Jensen, 1974 ; Pépin, 2003). Cependant, ces lésions pouvaient aussi être causées par Staphylococcus aureus subsp. anaerobius (Euzeby, 1999 ; Moller et al., 2000 ; Sayed et al., 1995). Des lésions de nécrose et de suppuration diffuses et sévères observées dans $13,3 \%$ des cas reflétaient un pouvoir pathogène marqué des germes responsables (De La Fuente et al., 1985 ; Pépin et al., 1991 ; Pépin, 2003 ; Sayed et al., 1995). En effet, certaines souches de C. pseudotuberculosis et de $S$. aureus ont une virulence marquée responsable de lésions sévères de nécrose lymphocytaire (Euzeby, 1999).

Des abcès ont déjà été rapportés chez les ovins de la race D'man dans les régions du sud-est du Maroc avec une prévalence de 24,5\% (Ait Balahcen, 2000) et ont été attribués à S. aureus subsp. anaerobius (61,5\%), à Streptococcus dysgalactiae (9,6\%), à Actinomyces pyogenes $(7,7 \%)$ et à $C$. pseudotuberculosis $(5,6 \%)$. En outre, des abcès ont été décrits chez le dromadaire au sud du Maroc avec une prévalence de $18 \%$ (Ramiche, 2001), et sous forme de lésions de lymphadénite (68\% des cas) associées à $S$. aureus, $C$. ulcerans, $C$. pseudotuberculosis et/ou Streptococcus dysgalactiae, et de lésions cutanées (32\% des cas) liées principalement à S. aureus.

Les lésions observées au cours de notre étude étant similaires à celles décrites lors d'infection par C. pseudotuberculosis ou S. aureus subsp. anaerobius, il est nécessaire d'établir un diagnostic étiologique sûr en procédant à l'isolement et à l'identification des bactéries pathogènes à partir des abcès ganglionnaires, et à la caractérisation éventuelle de leur pouvoir pathogène. Un programme de lutte contre la maladie s'appuyant sur la prophylaxie médicale par la vaccination pourrait être envisagé (Erganiş et al., 2014 ; Moussa et al., 2014 ; Windsor, 2011) en association avec la prophylaxie sanitaire (Pépin, 2003 ; Windsor, 2011) basée essentiellement sur la lutte contre les facteurs prédisposant à l'apparition de la LC, comme l'usage de matériel traumatisant dans les élevages ovins.

\section{REFERENCES}

Ait Balahcen M., 2000. Etude clinique et épidémiologique de la lymphadénite caséeuse chez les petits ruminants dans la région de Ouarzazate. Thèse Doct. Méd. Vét., Institut agronomique et vétérinaire Hassan II, Rabat, Maroc, 75 p.

Al-Gaabary M.H., Osman S.A., Oreiby A.F., 2009. Caseous lymphadenitis in sheep and goats: Clinical, epidemiological and preventive studies. Small Rumin. Res., 87: 116-121, doi: 10.1016/j. smallrumres.2009.10.008

Alloui M.N., Kaba J., Ayachi A., Alloui N., Herhoura K., Briese A., Clauss M., Springorum A., Hartung J., 2009. Risk factors of abscess disease in sheep and goat of Batna area (Algeria). In: Sustainable animal husbandry: prevention is better than cure, Vol. 1. In: Proc. 14th Int. Congr. ISAH, Vechta, Germany, 19-23 July 2009, 297-300

Al-Qarawi A., 2005. Physiopathological changes associated with abscesses in sheep at Al-Qassim region of Saudi Arabia. In: Proc. 6th Int. Sheep Veterinary Congress, Greece, 17-21 June 2005, 126-127

Baird G.J., Malone F.E., 2005. Control of ovine caseous lymphadenitis based on regular ELISA testing. In: Proc. 6th Int. Sheep Veterinary Congress, Greece, 17-21 June 2005, 136-137

Batey R.G., 1986. Lesions of the head in ovine caseous lymphadenitis (bacteria sheep). Aust. Vet. J., 63 (4): 131

Blood D.C., Henderson J.A, Radostits O.M., 1994. Veterinary Medicine, 5th Ed. Baillère Tindal, London, UK, 1763 p.

Brugère-Picoux J., 2004. Maladie (lymphadénite) caséeuse. In : maladies des moutons, $2^{\mathrm{e}}$ éd. France agricole, Paris, France, 62-65

Buxton A., Fraser G., 1984. Animal microbiology. In : Immunology, bacteriology, mycology, diseases of fish and laboratory methods, Vol. 1. Blackwell Science, 178-183

D’afonseca V., Moraes P.M., Dorella F.A., Pacheco L.G.C., Meyer R., Portela R.W., Miyoshi A., Azevedo V., 2008. A description of genes of Corynebacterium pseudotuberculosis useful in diagnostics and vaccine applications. Genet. Mol. Res., 7 (1): 252-260

De La Fuente R., Suarez G., Schleifer K.H., 1985. Staphylococcus aureus subsp. anaerobius, the causal agent of abscess disease of sheep. Int. J. Syst. Bacteriol., 35: 99-102, doi: 10.1099/00207713-35-1-99

Erganiş O., Hadimli H.H., Kav K., Sakmanoğlu A., Sayin Z., Pinarkara Y., 2014. Efficacies of Corynebacterium pseudotuberculosis vaccines against caseous lymphadenitis in mice and sheep. Eurasian J. Vet. Sci., 30 (2): 72-79, doi:10.15312/EurasianJVetSci.201425922

Euzeby J., 1999. List of prokaryotic names with standing in nomenclature. Immunology, bacteriology, mycology, diseases of sheep and laboratory methods. Blackwell Science, 178-183

Gironés O., Simon M.C., Alonso J.L., 1992 Linfadenitis caseosa. I. Importancia econômica-sanitaria. Etiologia, epidemiologia y patogenia. Med. Vet., 9: 135-148

Jensen R., 1974. Caseous lymphadenitis (pseudotuberculosis). Diseases of sheep. Lea \& Febiger, Philadelphia, PA, USA, 366-369

Kichou F., Tligui N., Najjari A., Drissi M., 2003. Dominantes pathologiques chez les caprins du Nord marocain : Cas de la région de Tetouan. Actes Inst. Agron. Vet. (Maroc), 23 : 73-79

Malone F.E., Fee S.A., Kamp E.M., King D.C., Baird G.J., Reilly K.M., Murdock F.E.A., 2005. A comparison of pathological, bacteriological and serological examinations in sheep from four flocks naturally infected with caseous lymphadenitis. In: Proc. 6th Int. Sheep Veterinary Congress, Greece, 17-21 June 2005, 226-227

Moller K., Agerholm J.S., Ahrens P., Jensen N.E., Nielsen T.K., 2000. Abscess disease, caseous lymphadenitis, and pulmonary adenomatosis in imported sheep. J. Vet. Med B., 47: 55-62

Moussa I.M., Hessain A.M., Hemeg H.A., Selim S.A., 2014. Single-point mutation as a molecular tool for preparation of recombinant vaccine against caseous lymphadenitis. J. Food Agricul. Environ., 12 (2): 626629

Mubarak M., Bastawrows A.F., Abdel-Hafeez M.M., Ali M.M., 1999 Caseous lymphadenitis of sheep and goats in Assiut farms and abattoirs. Asst. Vet. Med. J., 42: 89-112 
Oreiby A.F., Hegazy Y.M., Osman S.A., Ghanem Y.M., Al-Gaabary M.H. 2014. Caseous lymphadenitis in small ruminants in Egypt. Clinical, epidemiological and prophylactic aspects. Tierärztl. Prax., 42 (G): 271-277

Paton M.W., Mercy A.R., Sutherland S.S., Ellis T.M., 1988. The influence of shearing and age on the incidence of caseous lymphadenitis in Australian sheep flocks. Acta Vet. Scand., 84 (suppl.): 101-103

Paton M.W., Rose I.R., Hart R.A., Sutherland S.S., Mercy A.R., Ellis T.M., Dhaliwal J.A., 1994. New infection with Corynebacterium pseudotuberculosis reduces wool production. Aust. Vet. J., 71: 47-49, doi: 10.1111/j.1751-0813.1994.tb06152

Paton M.W., Walker S.B., Rose I.R., Watt G.F., 2003. Prevalence of caseous lymphadenitis and usage of caseous lymphadenitis vaccines in sheep flocks. Aust. Vet. J., 81: 91-95, doi : 10.1111/j.17510813.2003.tb11443

Pépin M., 2003. La lymphadénite caséeuse. In : Principales maladies infectieuses et parasitaires du bétail - Europe et régions chaudes. Tome 2 : Maladies bactériennes, mycoses et maladies parasitaires (coord Lefèvre P.-C., Blancou J., Chermette R.). Editions Tec \& Doc, Lavoisier, Cachan, France, 1007-1019

Pépin M., Fontaine J.J., Pardon P., Marly J., Parodi A.L., 1991 Histopathology of the early phase during experimental Corynebacterium pseudotuberculosis infection in lambs. Vet. Microbiol., 29 (2): 123-134, doi: 10.1016/0378-1135(91)90120-5
Pépin M., Pardon P., Marly J., Lantler F., 1988. Corynebacterium pseudotuberculosis infection in adult ewes by inoculation in the external ear. Am. J. Vet. Res., 49: 459-463

Pépin M., Paton M., Hodgson L.M., 1994. Pathogenesis and epidemiology of Corynebacterium pseudotuberculosis infection in sheep. Curr. Trop. Vet. Res., 1: 63-82

Pépin M., Sanchis R., Paton M., 1999. La lymphadénite caséeuse des ovins et des caprins. Point. Vét., $30: 33-40$

Ramiche A., 2001. Etude des abcès superficiels chez le dromadaire (Camelus dromedarius) dans le sud du Maroc. Thèse Doct. Méd. Vét., Institut agronomique et vétérinaire Hassan II, Rabat, Maroc, 140 p.

Sayed A.M., Abdel-Fattah A.M., Manaa A.M., 1995. Caseous lymphadenitis of sheep in Assiut governorate: disease prevalence, lesion distribution, and bacteriological. Assiut Vet. Med. J., 33: 88-92

Shreuder B.E.C., Ter Laak E.A., Griesen H.W., 1986. An outbreak of caseous lymphadenitis in dairy goats: First report of the disease in the Netherlands. Vet. Q., 8: 61-67, doi: 10.1080/01652176.1986.9694019

Smith M.C., 1981. Caprine dermatology problems: a review. J. Am. Vet. Med. Ass., 178: 724

Windsor P.A., 2011. Control of caseous lymphadenitis. Vet. Clin. North Am. Food Anim. Pract., 27: 193-202, doi: 10.1016/j.cvfa.2010.10.019

\section{Summary}

Kichou F., Mechaal A., Bouslikhane M., Kadiri A., Zro K., Berrada J. Risk factors and clinical and lesion characteristics of caseous lymphadenitis or abscess disease in sheep in Morocco

The aims of this study were to determine the prevalence and risk factors of caseous lymphadenitis in sheep in the Oriental region in Morocco, and to determine its clinical and lesion characteristics. Surveys were conducted in different provinces of the region. They focused on 6376 animals in 107 farms. In addition, search for lesions, and lymph node and abscess collection for histopathological examination were performed in five slaughterhouses of the region. The results showed that almost $100 \%$ of the farms (106 out of 107) were affected by caseous lymphadenitis. The overall individual prevalence of the disease was $24 \%$, and the average prevalence in farms and slaughterhouses were 28 and $16 \%$, respectively. Besides the poor hygiene and high density of sheep in houses, use of traumatic equipment (metal feeders, drinkers and fences) seemed to be the main factor that lead to the spread of abscesses between animals of a same herd. Superficial abscesses, open or not, surrounded by enlarged lymph nodes were present in all affected animals. The location of the lesions varied but about $79 \%$ of them were located in the head or the neck. Histopathological examination of lymph nodes revealed two types: active abscesses (87\%), and lesions of diffuse and severe necrosis and suppuration of the lymphoid tissues $(13 \%)$. In the lungs, abscesses were well defined and circumscribed.

Keywords: sheep, caseous lymphadenitis, abscess, risk factor, histopathology, epidemiology, Morocco

\section{Resumen}

Kichou F., Mechaal A., Bouslikhane M., Kadiri A., Zro K., Berrada J. Factores de riesgo y características clínicas y de las lesiones de linfadenitis caseosa o enfermedad de los abscesos en ovejas en Marruecos

Los objetivos del presente estudio fueron de determinar la prevalencia y los factores de riesgo de la linfadenitis caseosa en ovejas en la región oriental en Marruecos, y determinar las características clínicas y de las lesiones. Se llevaron a cabo encuestas en diferentes provincias de la región. Se enfocaron en 6376 animales en 107 fincas. Adicionalmente, se Ilevaron a cabo búsquedas de lesiones, y colecta de linfonodos y de abscesos para examen histopatológico en cinco mataderos de la región. Los resultados muestran que casi $100 \%$ de las fincas (106 de 107) se vieron afectadas por la linfadenitis caseosa. La prevalencia individual general de la enfermedad fue de $24 \%$ y la prevalencia promedio en fincas y mataderos fue de 28 y $16 \%$ respectivamente. Aparte de la poca higiene y alta densidad de ovejas en las casas, el uso de equipo traumático (comederos, bebederos y cercas de metal) pareció ser el principal factor que llevo a la diseminación de abscesos entre los animales de un mismo hato. Abscesos superficiales, abiertos o no, con linfonodos agrandados, estaban presentes en todos los animales. La localización de las lesiones varió, pero alrededor de $79 \%$ estaban localizadas en la cabeza o el cuello. El examen histopatológico de los linfonodos reveló dos tipos: abscesos activos (87\%) y lesiones de necrosis difusa y severa y supuración de los tejidos linfoides (13\%). En los pulmones, los abscesos estaban bien definidos y circunscritos.

Palabras clave : ovino, linfoadenitis caseosa, absceso, factor de riesgo, histopatología, epidemiología, Marruecos 